\title{
EVALUATION OF CHONDROPROTECTIVE EFFECTS OF INTRA ARTICULAR PIROXICAM IN POST-TRAUMATIC OSTEOARTHRITIS MODEL OF RAT
}

\author{
Noaman Ishaq, Shabana Ali*, Qurra Tul Ain Haider*, Muhammad Abdul Basit Qaisrani**, Komal Mumtaz Malik*, \\ Muhammad Wasiullah Khan* \\ Bakhtawar Amin Medical and Dental College, Multan Pakistan, *Army Medical College/National University of Medical Sciences (NUMS) Rawalpindi Pakistan, \\ **THQ Karor Lal, Layyah Pakistan
}

\begin{abstract}
Objective: To evaluate the chondroprotective effect of piroxicam in post-traumatic osteoarthritis model of rat. Study Design: Laboratory based experimental study.

Place and Duration of Study: Pharmacology department, Army Medical College, Rawalpindi, from Apr to Jun 2019.

Methodology: Project included sixteen rats of Sprague Dawley breed. Osteoarthritis was induced in anesthetized rats by surgical removal of medial meniscus and anterior cruciate ligament resection. After that rats were randomly allocated in two groups with eight rats in each group. Rats of group I were positive control that received $0.2 \mathrm{ml}$ saline intra articularly once weekly for four weeks. Meanwhile rats of group II (treatment group) received $50 \mu 1$ piroxicam intra articularly once weekly for four weeks. One week after the drug intervention, radiograph of the right knee joint of all rats were taken. Animals were then sacrificed with inhaled chloroform and part of proximal tibia was obtained for histopathological analysis.

Results: Comparison of radiographs of both groups depicted a significant $p$-value of $<0.01$. Meanwhile mean histopathological score of control group and treatment group were $11.50 \pm 1.195$ and $6.50 \pm 1.195$ respectively with a $p$-value of $<0.01$.

Conclusion: Intra articular administration of piroxicam in post-traumatic Osteoarthritis model of rats resulted in improvement in radiographic grades and histopathology scores.
\end{abstract}

Keywords: Chondroprotective effects, Osteoarthritis, Piroxicam.

This is an Open Access article distributed under the terms of the Creative Commons Attribution License (http://creativecommons.org/licenses/by/4.0), which permits unrestricted use, distribution, and reproduction in any medium, provided the original work is properly cited.

\section{INTRODUCTION}

Osteoarthritis (OA) was one of the conspicuous causes of mobility-related disability. Age related degenerative changes are the foremost cause of development of OA in older people. Meanwhile trauma to affected joint is the leading cause of development of $\mathrm{OA}$ in people younger than 45 years of age ${ }^{1}$. Young person who sustains a joint injury are recognized to be at significantly increased risk of developing OA as compared to non-injured one. OA that progresses after joint injury is termed as post-traumatic OA (PTOA). Individuals with PTOA approximately accounts $12 \%$ of all cases symptomatic $\mathrm{OA}^{2}$. Risk of PTOA is more common in younger healthier and more active people as compared to idiopathic OA which is common in old age. That's why patient population suffering from PTOA requires medical and surgical intervention at a much earlier age in life. Knee joint are the the most vulnerable joint for developing $\mathrm{PTOA}^{3}$. Any injury severe enough to cause anterior cruciate ligament (ACL) tear or meniscus destabilization frequently leads to PTOA in knee joint ${ }^{4}$. PTOA affects whole joint architecture including bone, ligaments, bursas, synovium and

Correspondence: Dr Noaman Ishaq, Pharmacology Department, Bakhtawar Amin Medical and Dental College, Multan Pakistan Received: 24 Jun 2020; revised received: 29 Oct 2020; accepted: 11 Nov 2020 periarticular muscles. Non pharmacological measures that use to manage PTOA is awareness and education of patient about disease pathology and to persuade him to avoid triggering factors of disease progression ${ }^{5}$. Meanwhile drug groups are used to lessen the severity of symptoms and to delay the progression of disease. Nonsteroidal anti-inflammatory drugs (NSAIDs), corticosteroids and viscosupplement substances are widely used drug groups in the treatment of PTOA ${ }^{6}$. Piroxicam is one of the investigational NSAID that is commonly prescribed to patients of PTOA. It not only relieves pain but also subsides inflammatory component of PTOA. Long duration of action is the advantage of Piroxicam to many other NSAIDs. Piroxicam is a non selective reversible inhibitor of COX enzyme. It competes with arachidonic acid to bind with COX enzyme. Inhibition of COX enzyme leads to impaired generation of prostaglandins (PGs), prostacyclins and thromboxanes. COX I enzyme is present mainly in the stomach while Prime location of COX II is the site of inflammation and tissue injury. Inhibition of COX I causes disruption of physiological process of gastric protection that leads to GI symptoms ranging from mild dyspepsia to erosion and rarely severe bleeding. Similarly inhibition of COX II leads to decrease concentration of PGE1 and PGF2a in inflamed tissues that not only relieves pain but also lessens inflammation. 
To avoid gastrointestinal symptoms of oral therapy as well as to obtain targeted effects of the drug, Intra articular (IA) route of drug administration is practiced. IA administration of therapeutic agents has been clinically explored to selectively deliver active compounds to their site of action in the treatment of osteoarthritis, rheumatoid arthritis, and joint pain. Direct IA delivery of active compounds to affected tissues offers the chance to boost therapeutic outcomes with lower dose, while reducing systemic exposure and undesirable adverse effects. Beside COX inhibition, Piroxicam reduces the vasodilation property of vessels in response to bradykinin and histamine. Thus it also reverses the vasodilation of inflammatory tissues. It also lessens lymphokines production from $\mathrm{T}$ lymphocytes. Recent studies proposed that Piroxicam is also involved in inhibition of central component of pain mediation. It blocks the B2 receptor mediated pathway in the sensory neurons and thus inhibits bradykinin induced nociception 7,8 .

Rationale of this animal study was as Piroxicam is an investigational drug in the management of PTOA, we planned this project to detect chondroprotective effects of IA piroxicam in a rat model of PTOA.

\section{METHODOLOGY}

It was a laboratory based experimental study that was carried out in department of Pharmacology and Therapeutics, Army Medical College (AMC), Rawalpindi in collaboration with National Institute of Health (NIH), Islamabad. This study was ethically approved from ethics review committee of "Centre for Research in Experimental and Applied Medicine (CREAM)", AMC. Intervention protocol of this study was about two months from April 2019 to June 2019. Rats were kept and nurtured in animal house of NIH during the complete study period. Preliminary sixteen (16) adult male or non-pregnant female rats of Sprague Dawley breed, of about 02 months and weighing about 400 gram were selected through non probability consecutive sampling. Conventional cages with wood chip bedding were used and animals were identified by a study period ${ }^{9}$. They were allocated randomly in two (02) groups with eight (08) rats in each group. Surgical procedure was performed to induce changes similar to PTOA in right knee joint of all rats. They were anesthetized with intraperitoneal injection of $5 \%$ xylazine and $1 \%$ ketamine $^{10}$. Skin of the joint was shaved with sterile razor and disinfected with pyodine followed by a para patellar incision on medial side of the joint. Skin and fascias were retracted for the complete exposure of joint. After identification anterior cruciate ligament was removed and medial meniscus was transected. Skin was closed with surgical stapler thereafter. Later on Animals were permitted to move freely in the cage for two weeks ${ }^{11}$. Then intra articular drugs were administered in the right knee joint of the rats. Rats of control group and treatment groups were injected with 0.2 $\mathrm{ml}$ of Normal saline and $70 \mu \mathrm{l}(1.4 \mathrm{mg} / \mathrm{ml})$ piroxicam once weekly for 04 weeks ${ }^{12}$. We delayed for one week more and then radiographs of corresponding joint of anesthetized (with 5\% xylazine and 1\% ketamine) rats were taken and graded according to Kellegren and Lawrence grading system with the help of radiologist ${ }^{13}$. After that animals were sacrificed with inhaled chloroform in a desiccator and sample of proximal tibia was obtained by using 5.5"angled bone cutter ${ }^{14}$. After histological slide preparation, these slides were scored using Modified Mankin slide preparation ${ }^{15}$. Obtained data was statistically analyzed using SPSS version 25 . Radiographic grades were qualitative parameter and it was compared with Fischer Exact test while histopathological score was quantitative parameters and compared through student t-test. The differences between two observations were considered statistically significant if the $p$-value was equal or less than $0.05(p \leq 0.05)$.

\section{RESULTS}

The rats remained alive healthy and active throughout the study period. One week after the last IA injection Radiographs of right joint of anesthetized rats were taken and graded with the help of radiologist. Radiographs of 02 rats of control group had features of large osteophytes, marked joint space narrowing (JSN)

Table-I: Kellegren and Lawrence grading of rat model of Post traumatic osteoarthritis.

\begin{tabular}{l|c|c|c|c|c|c}
\hline \multirow{2}{*}{ Groups } & \multicolumn{5}{|c|}{ Grades } & \multirow{2}{*}{$\boldsymbol{p}$-value } \\
\cline { 2 - 7 } & Grade 0 & Grade 1 & Grade 2 & Grade 3 & Grade 4 & $2(25 \%)$ \\
\hline Group I (control group) & - & - & $2(25 \%)$ & $4(50 \%)$ & $<0.01$ \\
\hline Group II (piroxicam group) & - & $4(50 \%)$ & $4(50 \%)$ & - & - & \\
\hline
\end{tabular}

cage label. Environment of rats was maintained with temperature ranged $25 \pm 5^{\circ} \mathrm{C}$ and 12 hours day night cycle. Free excess to clean drinking water and standard rodent diet adlibitum were provided during the whole and severe sclerosis and their grade was 04. Meanwhile 04 rats of this group graded with grade 03 because multiple osteophytes, definite JSN and visible sclerosis were features of their radiographs. Two rats 
of control group depicted definite osteophytes with possible JSN and grade of their radiograph was 2. Fig-1 is an X-ray of a rat of control group with grade 03 . Meanwhile radiograph of half rats of piroxicam group showed characteristics of doubtful JSN and possible osteophyte lipping and their grade was 01 and radiographs of other half of rats of piroxicam group exhibited features of definite osteophytes and possible JSN and their grade was 02. Figure-2 was a photomicrograph of rat of piroxicam group with minimal changes of OA and its grade was 01. Table-I is a cross tabulation of radiographic grades of both group. When these radiographs were compared with fischer exact test, we found significant $p$-value of $<0.01$ that explained that intra articular piroxicam exhibited chondroprotective effects in PTOA model of rat at radiological level.

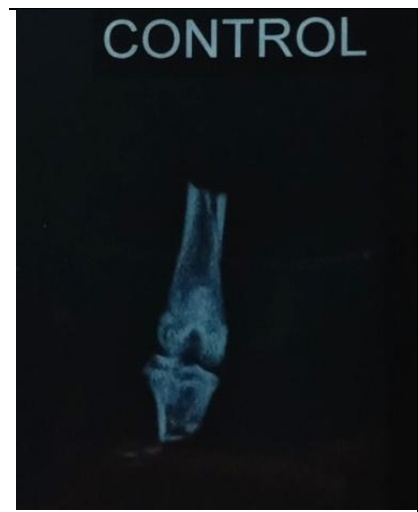

Figure-1: X-ray of rat of control group.

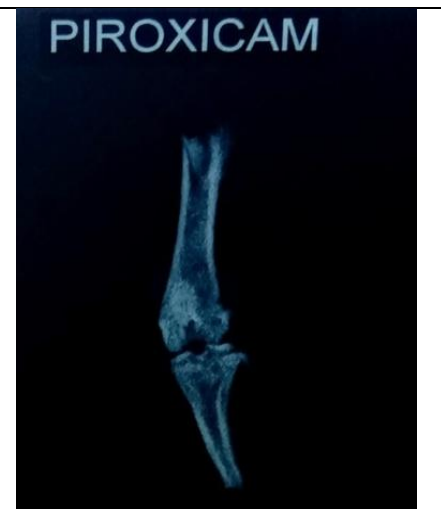

Figure-2: X-ray of rat of piroxicam group histopathology.

Histopathological analysis of all slides of both group was done under X100 and X400 lens. These histopathological changes were scored according to Modified Mankin scoring system.

Score of slides of control group was in range of 10-13. Six out of eight slides showed marked while rest of two showed mild irregularity in perichondrium. One slide had marked, six had moderate and one had mild fibrosis of perichondrium. Six slides had moderate to mark while two slides had mild to moderate irregularity of organization. All slides showed moderate to marked increase in cellularity of chondrocytes. Five slides showed moderately increased while three slides showed mild increase in chondrocyte clusters. Five slides showed $10-20 \%$ while three slides showed $20-80 \%$ necrosis of chondrocytes. Fibrinoid degeneration was feature of all the slides of disease control group. Figure- 3 is photomicrograph of slide of rat of this group with 13 score. Mean score of group I was $11.50 \pm 1.195$.
Slides of group II (piroxicam group) scored around 5-8. Mild irregularity of perichondrium was the feature of all slides. One had none, six had mild, while one had moderate fibrosis of perichondrium. Seven had mild to moderate while one had moderate to marked irregularity in organization. One slide showed no, four slides showed mild and three slides showed moderate to marked increase in cellularity of chondrocytes. Seven slides showed mild while one slide showed moderate increase in chondrocyte cluster. 10$20 \%$ necrosis of chondrocyte was the feature of all slides. None of the slides exhibited fibrinoid degene-

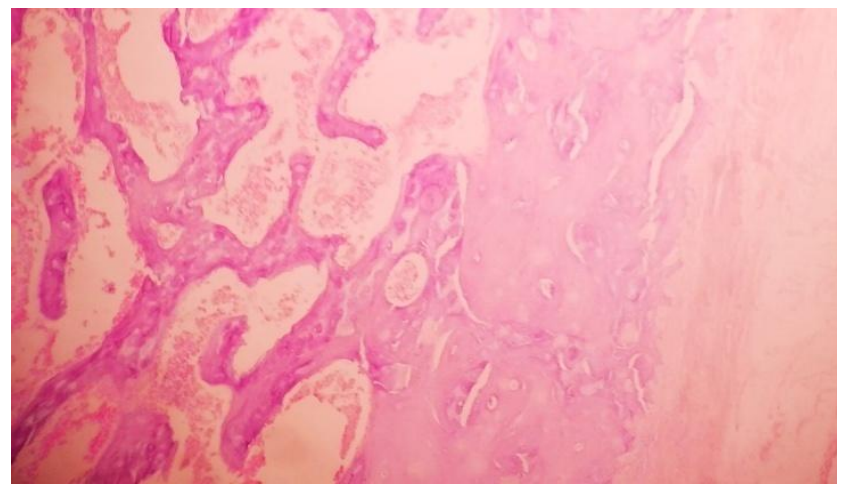

Figure-3: Photomicrograph of proximal tibia of a rat of group I (Control group).

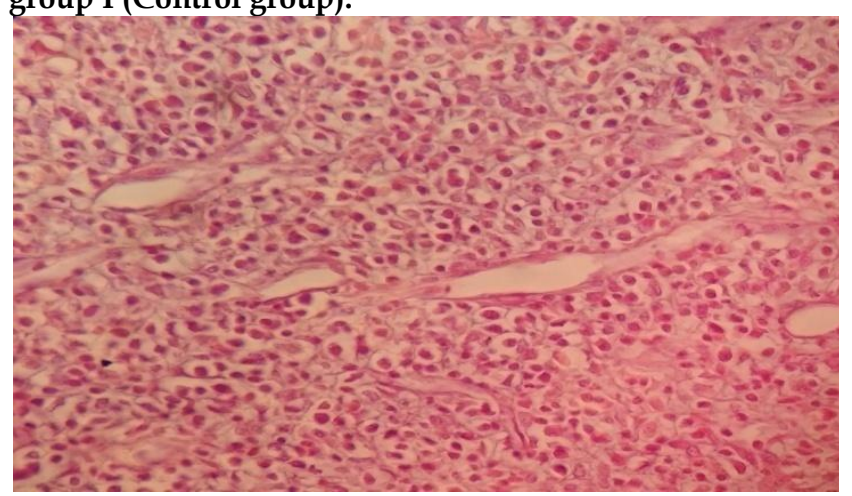

Figure-4: Photomicrograph of proximal tibia of a rat of group II (Piroxicam group).

ration. Figure-4 is a photomicrograph of slide of rat of piroxicam group with 07 score. It showed features of mild perichondrium fibrosis, moderate irregularity in organization, hypercellularity and $10-20 \%$ of chondrocytes necrosis. Mean score of this group was $6.50 \pm$ 1.195. When the histological score of both groups were compared with student $t$-test, we found a significant $p$ value of $<0.01$.

In short we observed a visible decrease of Kellegren and Lawrence radiographic grades and Mean Modified Mankin score of piroxicam group as compared to control group. Inter group comparison of radio- 
graphic grades and histopathological score exhibited the $p$-value $<0.01$ both times that confirmed the chondroprotective efficacy of piroxicam in rat model of PTOA.

\section{DISCUSSION}

Nonsteroidal anti-inflammatory drugs (NSAIDs), corticosteroids and viscosupplement substances are group of drugs that are frequently used in the management of post-traumatic osteoarthritis (PTOA) ${ }^{16}$. Piroxicamis an oxicam derivative NSAID that is frequently prescribed in pain conditions including PTOA all over the world. This animal study was considered to find out the chondroprotective effects of intra articular (IA) piroxicam in PTOA rat model. Human admissible dosage of piroxicam that was proficiently effective in rat model was selected by substantial search and literature review. After intervention protocol, when radiographic grades and modified mankin score were compared, differences between rats of piroxicam group and disease control group were statistically significant that confirmed the chondroprotective effects of piroxicam. A similar results found in studies of Park and his colleagues. It has proven in their study that there was a statistically significant difference $(p<0.05)$ of joint swelling and PGE2 level in IA piroxicam treated rats as compared to IA saline treated rat models of $\mathrm{OA}^{17}$. Likewise research work of Aziza and Hana revealed that IM therapy of piroxicam reduces joint edema and arthritic index statistically significantly $(p<0.05)$ in Freud Adjuvant induced arthritis models of rat ${ }^{18}$. Their findings also strengthen our results regarding chondroprotective efficacy of piroxicam. Meanwhile Research work of Ijaz ul Haq and his colleagues also proved chondroprotective efficacy of piroxicam $(p<0.05)$ in rabbit models of OA that also reinforce our results ${ }^{19}$.

Tenoxicam, an oxicam drug just like piroxicam, used intra articularly in the treatment of surgically induced PTOA rat model. Histological analysis depicted a significant $p$-value of 0.028 when compared Tenoxicam treated rats with non treated rats. Thus it was claimed that Tenoxicam has chondroprotective effects also signifying our target drug piroxicam might have same chondroprotective effects against $\mathrm{OA}^{20}$. Likewise meloxicam, another oxicam derivative sister drug of piroxicam, is used in chemically induced rat model of OA. It significantly decreased $(p=0.02)$ heat stimulation of affected joint as well as TNF-a level as compared to control group that also strengthened our results ${ }^{21}$.

This study also has some curbs. Though IA route of piroxicam administration leads to less systemic exp- osure, we did not study the adverse effects. Future studies should be explored that also observe the systemic adverse effects. Similarly a study alike to this contemporary study should be accompanied with prolonged duration between surgical trauma and drug administration. Delay after trauma of up to four to eight weeks may lead to more severe form of disease ${ }^{22}$.

\section{ACKNOWLEDGEMENT}

Authors are grateful to Dr. Hussain Ali, Head of Research animal facility, National Institute of Health, Islamabad for his supervision regarding induction of $\mathrm{OA}$ and IA drug administration in rat model. A special thanks to Army Medical College, Rawalpindi, for facilitating us to carried out this research project.

\section{Disclosure}

We are highly thankful to National University of Medical Sciences, Rawalpindi for funding our project.

\section{CONCLUSION}

IA administration of $70 \mu \mathrm{l}(2 \mathrm{mg} / \mathrm{ml})$ piroxicam once weekly for 04 weeks lead to reduced radiographic grades and mean Modified Mankin score of histopathology as compared to rats treated with saline only. Thus we concluded that piroxicam exhibits chondroprotective efficacy in PTOA models of rats.

\section{CONFLICT OF INTEREST}

This study has no conflict of interest to be declared by any author.

\section{REFERENCES}

1. Loeser RF, Collins JA, Diekman BO. Ageing and the pathogenesis of osteoarthritis. Nat Rev Rheumatol 2016; 12(7): 412-20.

2. Thomas AC, Hubbard-Turner T, Wikstrom EA, Palmieri-Smith RM. Epidemiology of posttraumatic osteoarthritis. J Ath Train 2017; 52(6): 491-96.

3. Carbone A, Rodeo S. Review of current understanding of posttraumatic osteoarthritis resulting from sports injuries. J Orthop Res 2017; 35(3): 397-405.

4. Rai MF, Duan X, Quirk JD, Holguin N, Schmidt EJ, Chinzei N, et al. Post-traumatic osteoarthritis in mice following mechanical injury to the synovial joint. Sci Rep 2017; 7(1): 1-3.

5. Hall M, Castelein B, Wittoek R, Calders P. Diet-induced weight loss alone or combined with exercise in overweight or obese people with knee osteoarthritis: A systematic review and metaanalysis. Semin Arthritis Rheum 2019; 48(5): 765-77.

6. Bajpayee AG, Grodzinsky AJ. Cartilage-targeting drug delivery: Can electrostatic interactions help?. Nat Rev Rheumatol 2017; 13(3): 183-93.

7. Kim SR, Ho MJ, Kim SH, Cho HR, Kim HS. Inc-reased localized delivery of piroxicam by cationic nanoparticles after intraarticular injection. Drug Des Devel Ther 2016; 10(1): 3779-87.

8. Santenna C, Kumar S, Balakrishnan S. A comparative experimental study of analgesic activity of a novel non-steroidal anti-inflammatory molecule-zaltoprofen, and a standard drug-piroxicam, using murine models. J Exp Pharmacol 2019; 11(2): 85-91. 
9. Mus LM, Denecker G, Speleman F, Roman BI. Vehicle development, pharmacokinetics and toxicity of the anti-invasive agent 4-fluoro-3', 4', 5'-trimethoxychalcone in rodents. PloS One 2018; 13(2): 1-23.

10. Saadat E, Shakor N, Gholami M, Dorkoosh FA. Hyaluronic acid based micelle for articular delivery of triamcinolone, preparation, in vitro and in vivo evaluation. Int J Pharm 2015; 489(1-2): 218-25.

11. Tsai HC, Chen TL, Chen YP, Chen RM. Traumatic osteoarthritisinduced persistent mechanical hyperalgesia in a rat model of anterior cruciate ligament transection plus a medial meniscectomy. J P Res 2018; 11(1): 41-50.

12. Ozkan FU, Uzer G, Türkmen I, Yildiz Y, Senol S, Ozkan K, et al. Intra-articular hyaluronate, tenoxicam and vitamin $\mathrm{E}$ in a rat model of osteoarthritis: evaluation and comparison of chondroprotective efficacy. Int J Clin Exp Med 2015; 8(1): 1018-26.

13. Madzuki IN, Lau SF, Tantowi NA, Ishak NI, Mohamed S. Labisiapumila prevented osteoarthritis cartilage degeneration by attenuating joint inflammation and collagen breakdown in postmenopausal rat model. Inflammopharmacology 2018; 26(5): 1207-17.

14. Asefi S, Seifi M, Fard GH, Lotfi A. Innovative evaluation of local injective gel of curcumin on the orthodontic tooth movement in rats. Dent Res J 2018; 15(1): 40-49.

15. Takahashi I, Matsuzaki T, Kuroki H, Hoso M. Joint unloading inhibits articular cartilage degeneration in knee joints of a monosodium iodoacetate-induced rat model of osteoarthritis. Osteo- arthritis Cartilage 2019; 27(7): 1084-93.

16. Rai SK, Raman VP, Varma R, Wani SS. Combined intra-articular injections (Hyaluronic acid, platelet-rich plasma, and corticosteroid) for osteoarthritis knee, an effective alternative treatment. J Orthop Traumatol Rehabil 2018; 10(1): 57-60.

17. Park CW, Ma KW, Jang SW, Son M, Kang MJ. Comparison of piroxicam pharmacokinetics and anti-inflammatory effect in rats after intra-articular and intramuscular administration. Biomol Ther 2014; 22(3): 260-66.

18. Amer AM, Mabrok H. Effect of joint inflammation on piroxicam pharmacokinetics in rats. Egypt J Vet Sci 2018; 49(2): 155-65.

19. Ijaz-Ul-Haq M, Khalid MU, Faheem M, Qasim S. Safety and efficacy assessment of different therapeutic regimens in experimentally induced osteoarthritis animal model. Env Pharmacol Life Sci 2018; 10(7): 109-19.

20. Ozkan FU, Uzer G, Türkmen I, Yildiz Y, Senol S, Ozkan K, et aI. Intra-articular hyaluronate, tenoxicam and vitamin $\mathrm{E}$ in a rat model of osteoarthritis: evaluation and comparison of chondroprotective efficacy. Int J Clin Exp Med 2015; 8(1): 1018-26.

21. Khotib J, Utami NW, Gani MA, Ardianto C. The change of proinflammatory cytokine tumor necrosis factor a level in the use of meloxicam in rat model of osteoarthritis. J Basic Clin Physiol Pharmacol 2019; 14(1): 126-36.

22. Bapat S, Hubbard D, Munjal A. Pros and cons of mouse models for studying osteoarthritis. Clin Transl Med 2018; 7(1): 36-48. 